\title{
Efecto de la inoculación con Rhizophagus irregularis y de la fertilización fosfatada sobre la comunidad local de hongos formadores de micorrizas arbusculares*
}

\section{Effect of inoculation with Rhizophagus irregularis and phosphate fertilization on the arbuscular mycorrhizal fungi local community}

\author{
ORDOÑEZ-CASTAÑEDA, YULI-MARCELA ${ }^{1}$; CEBALLOS-ROJAS, ISABEL-CRISTINA²; \\ RODRIGUEZ-VILLATE, ALIA ${ }^{3}$; SANDERS, IAN R. ${ }^{4}$
}

Historial del Artículo

Recibido para evaluación: 10 de Febrero 2021.

Aprobado para publicación: 24 de Abril 2021.

* Título del Proyecto de origen: "Cassava for food security and sustainability in Colombia: Biotechnological application of mycorrhizal fungi”. Financiación: Universidad Nacional de Colombia y Swiss National Science Foundation. Culminación: 01 de Septiembre de 2016.

1 Universidad Nacional de Colombia, Sede Bogotá. Facultad de Ciencias, Grupo de investigación Microbiología Agrícola. Magister en Ciencias. Bogotá, Colombia. https://orcid.org/0000-0001-8660-9869

2 Universidad Nacional de Colombia, Sede Bogotá. Facultad de Ciencias. Grupo de investigación Microbiología Agrícola. Doctor en Biotecnología. Bogotá, Colombia. https://orcid.org/0000-0002-0368-6349

3 Universidad Nacional de Colombia, Sede Bogotá. Facultad de Ciencias. Grupo de investigación Microbiología Agrícola. Doctor of Philosophy. Bogotá, Colombia. http://orcid.org/0000-0003-2167-0401

4 Universidad de Lausanne, Departamento de Ecología y Evolución. Grupo de investigación en ecología y evolución de organismos simbiontes. Doctor of Philosophy (Biology). Lausanne, Suiza. http://orcid.org/0000-0002-9591-8214 


\section{RESUMEN}

Los Hongos Formadores de Micorrizas Arbusculares (HFMA) son microorganismos cruciales en los suelos agrícolas por el efecto positivo que tienen sobre la movilización de uno de los nutrientes fundamentales para las plantas como es el fósforo (P). Los contenidos disponibles de P en los suelos de la Orinoquía Colombiana son bajos, por lo que la fertilización con fosfato es indispensable en cultivos comerciales como la yuca. El objetivo de este trabajo fue estudiar el efecto de la fertilización fosfatada y de la inoculación con Rhizophagus irregularis (hongo modelo de los HFMA), sobre las comunidades residentes de este grupo de hongos. La diversidad de los HFMA fue descrita a partir de las esporas presentes en la rizósfera de plantas de yuca mantenidas bajo condiciones agronómicas tradicionales. La riqueza y diversidad de las comunidades de HFMA se afectaron por la interacción de los factores analizados. En términos de estructura de la comunidad de HFMA en la rizósfera de las plantas de yuca, se detectaron cambios en la abundancia de los géneros Glomus y Acaulospora; asociados respectivamente a la inoculación y a la fertilización fosfatada. Los hallazgos de este estudio sugieren que prácticas agrícolas como la aplicación de fertilizante fosfatado y la inoculación con $R$. irregularis, son procesos que pueden afectar el ensamblaje de la comunidad de estos hongos, así también la diversidad de las comunidades de HFMA nativas en suelos de la Orinoquía Colombiana. Se requieren investigaciones adicionales para entender la relación entre la composición de las comunidades de HFMA y la respuesta de la planta hospedera.

\section{ABSTRACT}

Arbuscular Mycorrhizal Fungi (AMF) are important microorganisms in agricultural soils because of their positive effect on plant acquisition of phosphorus $(P)$; one of the fundamental nutrients for plants. Available $P$ in the soils of the Colombian Orinoquía are low, so phosphate fertilization is necessary in crops such as cassava. The objective of this work was to study the effect of phosphate fertilization and inoculation with Rhizophagus irregularis (the model AMF species), on the resident AMF communities. The AMF diversity was described by morphological and molecular techniques from the spores present in the rhizosphere of cassava plants maintained under traditional agronomic conditions. The richness and diversity of the AMF communities were affected by the experimental treatments AMF inoculation and P fertilization. Changes in abundance were detected in the genera Glomus and Acaulospora. This was significantly influenced by inoculation and phosphate fertilization. The findings of this study suggest that the use of phosphate fertilizer and the application of R. irregularis

\section{PALABRAS CLAVE:}

Hongos Formadores de

Micorrizas Arbusculares

(HFMA); Rhizophagus irregularis;

Diversidad; Manihot esculenta;

Fertilización Fosfatada; Inoculación; Comunidades de HFMA; Agricultura sostenible; Biofertilizantes; Esporas.

\section{KEYWORDS:}

Arbuscular Mycorrhizal Fungi (AMF); Rhizophagus irregularis; Diversity; Manihot esculenta; Phosphorus Fertilization; Inoculation; AMF Communities; Sustainable Agriculture; Biofertilizers; Spores.
Cómo citar este artículo: ORDOÑEZ-CASTAÑEDA, YULI-MARCELA; CEBALLOSROJAS, ISABEL-CRISTINA; RODRIGUEZ-VILLATE, ALIA; SANDERS, IAN R. Efecto de la inoculación Rhizophagus irregularis y de la fertilización fosfatada sobre la comunidad local de hongos formadores de micorrizas arbusculares. Biotecnología en el sector agropecuario y agroindustrial, v. 19, n. 2, 2021, p. 184-200. Doi: https://doi. org/10.18684/bsaa.v19.n2.2021.1850 
in agricultural processes affect the biodiversity of native AMF communities in soils of the Colombian Orinoquía. Further research is required to understand the relationship between AMF community composition and host plant response.

\section{INTRODUCCIÓN}

Los Hongos Formadores de Micorrizas Arbusculares (HFMA) recientemente reclasificados en el filo Mucoromycota, subfilo Glomeromycotina (Bonfante and Venice, 2020) son prevalentes en los ecosistemas terrestres y en suelos del neotrópico (Cofré et al., 2019; Stürmer and Kemmelmeier, 2021). La estimación del número de especies de este subfilo no ha sido completamente definida. Se han reportado hasta 244 especies de estos hongos basados en la morfología de esporas (Oehl et al., 2011) y hasta 338 especies tomando como referencia el análisis de ADNr ambiental o por secuencias amplificadas del gen ribosomal (Schussler, 2020). Los HFMA establecen simbiosis con más del $70 \%$ de las plantas terrestres (Brundrett and Tedersoo, 2018) y son un elemento fundamental en los agro-ecosistemas porque colonizan la mayoría de plantas de interés agronómico.

A nivel mundial, inóculos comerciales que incluyen propágulos de HFMA están disponibles para la industria agrícola y se han realizado diferentes experimentos para analizar su funcionalidad (Elliott et al., 2020; Frew, 2020). Con el uso de estos productos se incrementa la densidad de HFMA en el suelo para que actúen como biofertilizantes y así, promover la movilización de nutrientes que, al ser absorbidos por el micelio extra-radical, son transportados a la planta hospedera. La especie de HFMA más utilizada en la inoculación comercial es Rhizophagus irregularis. El uso de esta especie como inóculo en la práctica agrícola y también en estudios experimentales, se justifica por diferentes razones, por ejemplo, es una especie presente globalmente en diversos ecosistemas (Savary et al., 2018), puede colonizar de forma efectiva las raíces de la mayoría de las plantas (Brundrett and Tedersoo, 2018) y se establece con éxito después de su inoculación (Köhl et al., 2016).

La eficacia de estos inoculantes ha sido comprobada en cultivos de importancia mundial para la seguridad alimentaria, como son Manihot esculenta (Ceballos et al., 2013), Solanum tuberosum (Hijri, 2016) y Oryza sativa (Campo et al., 2020). Sin embargo es fundamental tener en cuenta que cada interacción inóculo-cultivo debe ser analizada de forma independiente y que la respuesta de la inoculación con HFMA depende de la variedad de la planta y del hongo que participan (Ceballos et al., 2019; García de León et al., 2020; Soonvald et al., 2020) En particular, para cultivos de yuca (M. esculenta) el beneficio de la inoculación ha sido demostrado en términos de productividad, es decir, en condiciones de campo y bajo prácticas agrícolas estándar. Así, en condiciones de cultivo comercial, en la Orinoquía Colombiana (Ceballos et al., 2013) y en Nigeria (Aliyu et al., 2019) se reportaron aumentos en el rendimiento de más del 20 y 12 \% respectivamente, cuando $R$. irregularis fue inoculado.

A la fecha y en términos de las comunidades locales de HFMA, no existe un concepto definitivo sobre las consecuencias ecológicas de utilizar un producto comercial que contenga una especie de estos hongos, por ejemplo R. irregularis, como inóculo en los sistemas agrícolas. Algunos estudios indican que la inoculación puede considerarse como un disturbio porque puede generar cambios en las comunidades pre-existentes de HFMA, con el reemplazo parcial (Koch et al., 2011) o completo de los hongos residentes (Jin et al., 2013). También se han reportado casos donde no se observó ninguna alteración (Renaut et al., 2020; Victorino et al., 2021), por lo tanto es difícil predecir el grado en el que la inoculación con HFMA puede alterar las comunidades residentes en un suelo (Hart et al., 2017). Adicionalmente, las evaluaciones reportadas sobre las consecuencias ecológicas de la inoculación, no se han realizado en sistemas experimentales en campo, ni tampoco en condiciones agrícolas comerciales del trópico en donde hay una enorme presión para aumentar la producción de alimentos con el fin de satisfacer la creciente población humana.

En los últimos años, para la descripción de las comunidades de HFMA asociadas a diferentes plantas de interés agronómico, se ha utilizado secuenciación masiva debido a las ventajas en términos de profundidad y cobertura de los análisis (Knerr et al., 2018; Song et al., 2019; Massa et al., 2020). Sin embargo, los estudios de HFMA a partir de esporas siguen siendo una estrategia fundamental dada la biología y genética de estos hongos. Se ha 
observado que hay un creciente interés en continuar los estudios de los HFMA utilizando los caracteres morfológicos y no exclusivamente, como fue la tendencia anterior, basados en secuenciación. Recientemente se han publicado investigaciones donde, con base en el reconocimiento y recuento de morfotipos (grupos de esporas similares morfológicamente), se describieron las comunidades micorrícicas (Ramírez-Gómez et al., 2019; Melo et al., 2020; Montenegro et al., 2020), se identificó el efecto que pueden tener la intensidad de labranza, la textura del suelo y el tipo de vegetación sobre las comunidades de HFMA (De la Cruz-Ortiz et al., 2020; Vieira et al., 2020); también se ha estudiado el efecto que tiene la deposición de nitrógeno y fósforo sobre la densidad de esporas de este grupo de hongos HFMA (Lin et al., 2020).

Los estudios publicados sobre la diversidad de los HFMA en suelos ácidos del trópico son escasos y se han centrado en la descripción de las comunidades sin abordar los cambios que pueden generar prácticas tradicionales agrícolas como, por ejemplo, la fertilización fosfatada. Los efectos del uso de fertilizantes sobre las comunidades de HFMA han sido estudiados en ecosistemas no agrícolas (Alguacil et al., 2010; Camenzind et al., 2014), sin embargo existe poca información de referencia sobre posibles alteraciones en la composición de las comunidades de HFMA, como consecuencia de la concentración de fósforo disponible en la rizósfera de las plantas de importancia agrícola (Séry et al., 2018). Esto es especialmente importante dado que se ha reportado una pérdida global de fósforo en suelos agrícolas (Alewell et al., 2020), que conlleva al uso estricto de fertilizantes fosfatados en la producción agrícola, más aún, en los suelos ácidos del trópico característicos de la Orinoquía Colombiana, y que típicamente presentan niveles bajos a muy bajos de este mineral.

En resumen, se requieren estudios sobre cambios en la diversidad de HFMA causados por la fertilización fosfatada y por el uso de biofertilizantes que contienen HFMA como $R$. irregularis, para entender el impacto que generan estas prácticas agrícolas sobre la comunidad de HFMA y así proporcionar información útil a los pequeños y grandes productores en la selección de inoculantes o de enmiendas inorgánicas sin alterar la biodiversidad.

En la presente investigación, se midió el disturbio causado por la fertilización fosfatada y la inoculación con Rhizophagus irregularis sobre la comunidad local de HFMA al final de un ciclo de cultivo, para lo cual se utilizaron métodos morfológicos y moleculares que permitieron describir las comunidades de esporas de HFMA asociadas a las plantas de yuca en la Orinoquía Colombiana. Previamente, se reportó el efecto de estas variable en términos de productividad agrícola, donde el mejor rendimiento del cultivo de yuca se alcanzó con la inoculación y utilizando fertilizante fosfatado al 50\% de lo aplicado tradicionalmente por los agricultores de la zona (Ceballos et al. 2013).

\section{MÉTODO}

\section{Diseño y establecimiento del experimento de campo}

La parcela experimental se instaló en la zona rural de Yopal, Casanare, Colombia, finca "Mate Pantano" de la Universidad de la Salle ( $72^{\circ} 17^{\prime} 48^{\prime \prime}$ W, $\left.5^{\circ} 19^{\prime} 31^{\prime \prime} \mathrm{N}\right)$. El terreno no había sido cultivado en los últimos 14 años y era una pastura en rastrojo. La variedad de yuca (Manihot esculenta Crantz) seleccionada fue MCOL2737 por ser una de las variedades más utilizadas por los agricultores de la zona y como inoculante se utilizó el producto comercial (Glomygel ${ }^{\circledR}$ Hortalizas (http://eigr.grupoei.com/i/i6833/index.php)) que contiene propágulos de $R$. irregularis.

Se estableció un diseño en bloques de dos factores y parcelas subdivididas. En cada uno de los cuatro bloques, la inoculación definió la parcela principal mientras que las parcelas subdivididas fueron marcadas por los tres niveles de fertilización fosfatada, para un total de seis tratamientos (Cuadro 1). Cada subparcela, contenía 24 plantas de yuca, sembradas a una distancia de $0,76 \mathrm{~m}$, donde las plantas tratamiento recibieron aleatoriamente un nivel de fertilización fosfatada: Sin fertilización (0 \% P), 50 \% P (100,5 g ha ${ }^{-1}$ fosfato diamónico) o 100 \% de fertilización (100 \% P, $201 \mathrm{Kg} \mathrm{ha}^{-1}$ fosfato diamónico). Las plantas no inoculadas y fertilizadas con $100 \%$ P representaron el manejo de un cultivo tradicional de yuca en la región. Alrededor de cada subparcela se sembraron dos filas de 
plantas de yuca para reducir el efecto borde. El manejo del cultivo fue convencional y no se requirió la aplicación de fungicidas. El impacto de la inoculación sobre el rendimiento del cultivo fue publicado previamente en Ceballos et al., (2013) por esta razón, detalles adicionales sobre la descripción del sitio de estudio, la implementación del experimento, el origen del material vegetal, entre otros, se encuentran publicados en ese estudio.

Cuadro 1. Tratamientos y variables en estudio.

\begin{tabular}{|c|c|c|}
\hline Número de tratamiento & Inoculación & Fertilizante Fosfatado \\
\hline 1 & SI & $0 \%$ \\
\hline 2 & SI & $50 \%$ \\
\hline 3 & SI & $100 \%$ \\
\hline 4 & NO & $0 \%$ \\
\hline 5 & NO & $50 \%$ \\
\hline 6 & NO & $100 \%$ \\
\hline
\end{tabular}

\section{Descripción de la comunidad de HFMA en el suelo rizosférico}

Tres plantas por cada bloque y tratamiento fueron seleccionadas aleatoriamente y muestras de suelo rizosférico (100 gramos) fueron colectadas al momento de la cosecha (12 meses después de la siembra), las cuales fueron secadas a temperatura ambiente y almacenadas a $4^{\circ} \mathrm{C}$ hasta su procesamiento. El suelo rizosférico por bloque y tratamiento fue agrupado en una muestra compuesta, para tener al final cuatro replicas por tratamiento. Para hacer un reporte cuantitativo de la diversidad de HFMA presente en el suelo rizosférico, se realizó la extracción de esporas en cada una de las muestras (INVAM, 2021a). Las esporas fueron clasificadas según su forma, color y tamaño en morfotipos. Se registró el número de morfotipos y la abundancia de cada morfotipo por muestra. Estos datos se utilizaron para calcular el índice de diversidad de Shannon y la riqueza de especies.

Se establecieron cultivos trampa para promover la multiplicación de los HFMA existentes en las muestras de suelo, con el objetivo de tener esporas jóvenes que favorecieran el análisis morfológico más detallado (INVAM, 2021b). Con las esporas provenientes de estos cultivos, se realizó un montaje en placa portaobjetos usando polivinil-lacto glicerol (PVLG) y reactivo de Melzer. Se hizo clasificación morfológica hasta nivel de orden y género cuando fuese posible, utilizando claves taxonómicas (Schenck and Perez, 1990). La caracterización a nivel de género se confirmó por métodos moleculares, para lo cual, entre dos a cinco esporas de cada morfotipo fueron agrupadas para la extracción de ADN (Sanders et al., 1995), sin el uso del Chelex ${ }^{\circledR}$ o sonicación. Las esporas fueron lavadas tres veces con agua destilada desionizada estéril $\left(\mathrm{H}_{2} \mathrm{O}\right.$-dde) y suspendidas en $20 \mu \mathrm{L} \mathrm{de} \mathrm{H}_{2} \mathrm{O}$-dde, se colocaron en tubos de polipropileno de $0,2 \mathrm{~mL}$ a $4^{\circ} \mathrm{C}$, y luego presionadas con mini-pistilos estériles hasta romperlas y liberar su contenido; $2 \mu \mathrm{L}$ de esta suspensión fueron colocados como templete directamente en la reacción de PCR utilizando 0,5 mM de cada cebador AML1-AML2 (Lee et al., 2008), 0,2 mM de dNTPs para una reacción con volumen final de $25 \mu \mathrm{L}$. El producto de la PCR fue diluido 1:100 y $2 \mu \mathrm{L}$ fueron usados como templete para la segunda PCR, donde se utilizó la pareja de cebadores NS31-AM1 (Helgason et al., 1998), con las siguientes condiciones: $94^{\circ} \mathrm{C}$ por 3 minutos, 30 ciclos de 1 min de denaturación a $95^{\circ} \mathrm{C}, 1$ min de hibridación de cebadores a $58^{\circ} \mathrm{C}$ y 1 min de extensión a $72{ }^{\circ} \mathrm{C}$, seguido por una extensión final de $72^{\circ} \mathrm{C}$ por $10 \mathrm{~min}$. Como control positivo se utilizó $A D N$ de $R$. irregularis extraído de un cultivo in vitro. El análisis filogenético se realizó utilizando las secuencias de la región SSU rDNA obtenidas en este estudio y tomando como base un árbol filogenético de referencia construido con las secuencias del gen ribosomal publicadas para el filo Glomeromycota (ahora subfilo Glomeromycotina) (1,5 kb, SSU-ITS-LSU) (Krüger et al., 2012), bajo el método RaxML-EPA (Senés-Guerrero et al., 2020)

\section{Análisis de diversidad}

La riqueza de especies (S'), el índice de diversidad de Shannon ( $\mathrm{H}^{\prime}$ ) y el Índice de Pielou (J'), fueron calculados para la comunidad extraradical de HFMA con base en la abundancia relativa de morfotipos. La distribución de los HFMA 
entre tratamientos fue observada utilizando escalamiento multidimensional no-métrico (NMDS), con los datos de abundancia relativa de cada morfotipo y sobre matrices de distancia Bray-Curtis. Estos datos fueron empleados también para el análisis de varianza multivariado permutacional (PERMANOVA) con el objetivo de comparar la composición de las comunidades entre tratamientos e identificar el efecto de la fertilización fosfatada y la inoculación. Los índices de diversidad alfa, así como NMDS y PERMANOVA fueron calculados utilizando las funciones 'estimateR', 'diversity', 'metaMDS' y 'adonis' en el paquete vegan (Oksanen et al., 2020) en R Core Team (2018).

\section{Análisis estadístico}

Los análisis estadísticos se realizaron utilizando el programa R versión 3.5.1 (R Core Team, 2018). Las diferencias entre tratamientos fueron encontradas significativas para un valor $p<0,05$ en todos los análisis. Para evaluar las diferencias estadísticas entre los índices de diversidad alfa causadas por la inoculación con $R$. irregularis y la fertilización fosfatada, se realizaron análisis de varianza (ANOVA) para el diseño en bloques al azar con arreglo en parcelas divididas utilizando los paquetes "agricolae" y "data.table". Para la comparación múltiple de medias de los índices de la diversidad alfa se utilizó la prueba Tukey, y la comparación entre la abundancia relativa de géneros de HFMA por tratamiento, fue realizada con la prueba de Kruskal-Wallis.

\section{RESULTADOS}

\section{Diversidad de HFMA en el suelo rizosférico de plantas de yuca en condiciones de campo}

En las muestras de suelo rizosférico de las plantas de yuca, se identificaron 37 morfotipos sobre los cuales se realizó la asignación taxonómica a nivel de género, de acuerdo con los resultados del análisis morfológico y molecular. Las secuencias obtenidas para 23 de los 37 morfotipos fueron depositadas en la base de datos del GenBank bajo los códigos consecutivos, desde MW486549 hasta MW486574 (https://bit.ly/3toqkqH ). Para los morfotipos restantes no se logró obtener una secuencia de ADN debido a que los amplicones no lograron clonarse, arrojaron un resultado en la secuenciación que no correspondía a Mucoromycota o las esporas aisladas no eran apropiadas para el estudio genético (esporas viejas o parasitadas); para estos casos, la asignación taxonómica se basó en las características morfológicas. De los 37 morfotipos identificados, 30 morfotipos se ubicaron en el orden Glomerales y los 7 restantes en el orden Diversisporales. A nivel de género, 18 morfotipos pertenecían a Claroideoglomus, siete a Rhizophagus, cinco a Glomus, seis a Diversispora y uno a Acaulospora. Morfotipos del género Claroideoglumus tuvieron la mayor proporción en la comunidad de esporas, para la mayoría de las muestras analizadas (Figura 1).

La comunidad de esporas de HFMA en todos los suelos analizados estuvo conformada por morfotipos de dos órdenes taxonómicos: Glomerales y Diversisporales. En promedio general, y teniendo en cuenta la información de todas las muestras analizadas, los Glomerales ocuparon mas del $80 \%$ de la comunidad extraradical, donde Claroideoglomus fue el género dominante y ocupó el $62 \%$, seguido del género Glomus y Rhizophagus. En el orden Diversisporales, Diversispora y Acaulospora estuvieron presentes en 10 y $3 \%$ respectivamente (Figura 2). Otros estudios. que describen la diversidad de comunidades de HFMA a partir de esporas en suelos del territorio colombiano, han reportado la dominancia de Glomerales en cultivos de plátano (Cabrales-Herrera et al., 2018), uchuva (Ramírez-Gómez et al., 2019) y en pasturas (Restrepo-Giraldo et al., 2019). Estudios en suelos de África también reportan la predominancia de Glomerales en la rizósfera de plantas de yuca (Séry et al., 2018). 


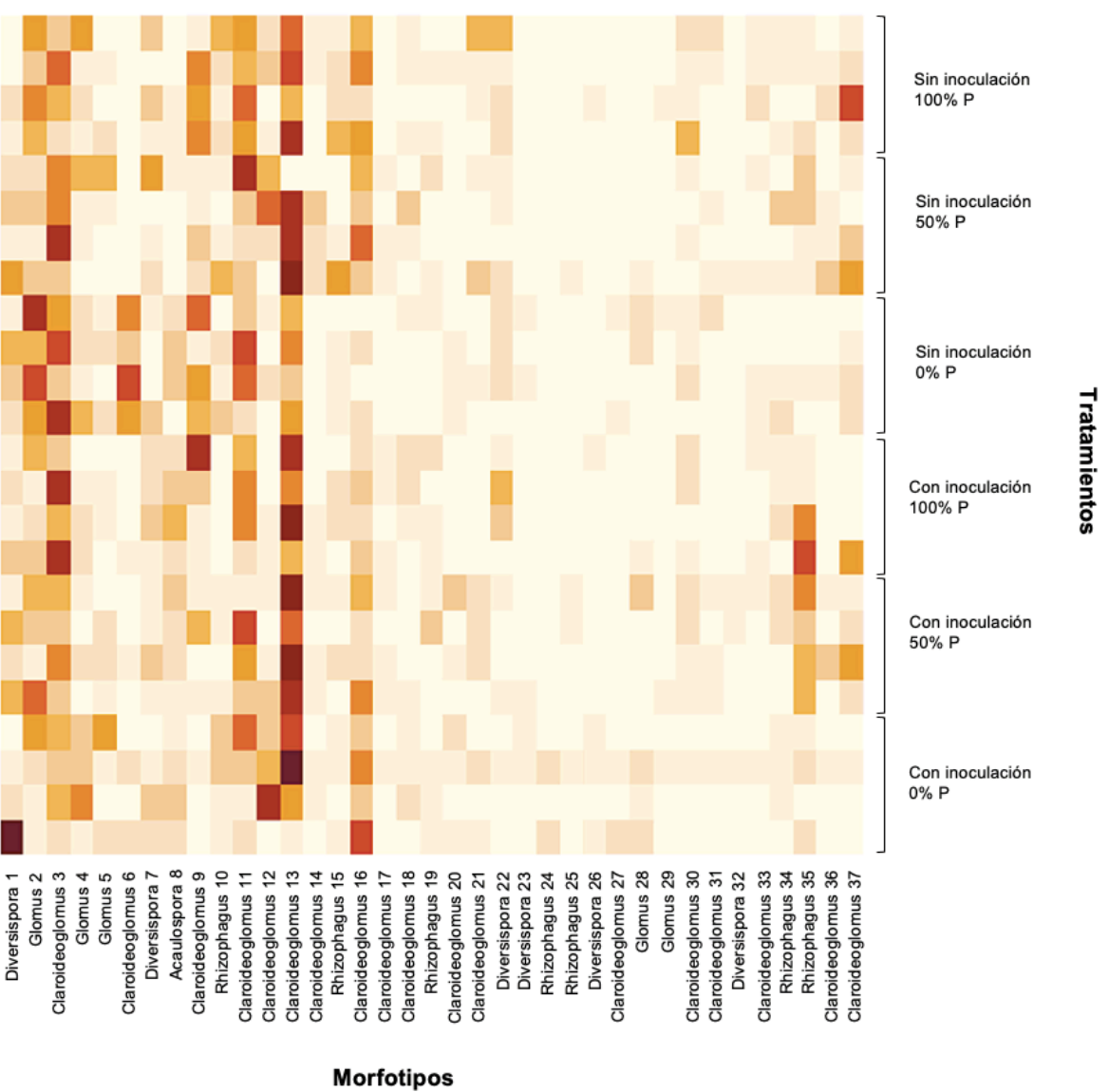

Figura 1. Mapa de calor que presenta la abundancia relativa de los morfotipos en cada una de las muestras analizadas, donde la intensidad del color se relaciona a una mayor abundancia.

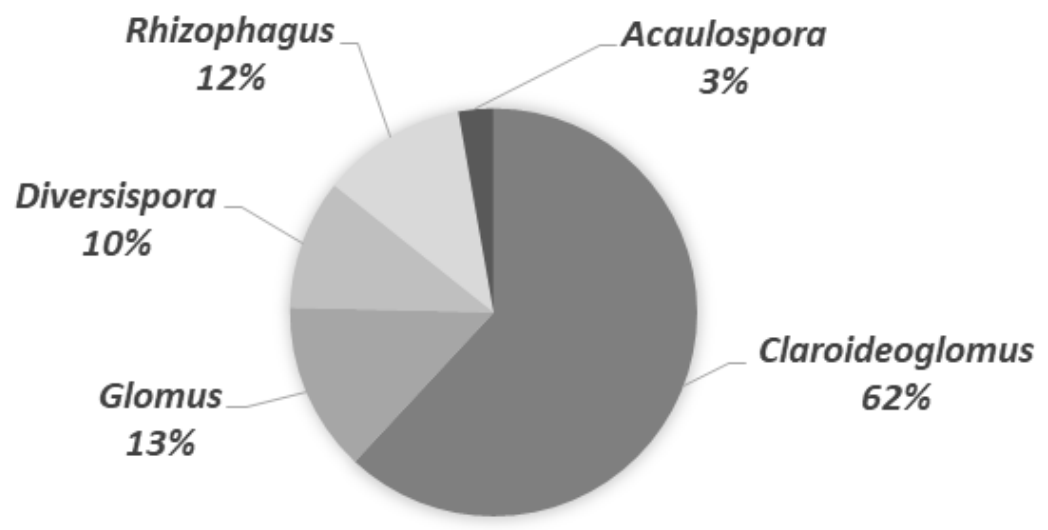

Figura 2. Distribución porcentual de los géneros que conformaron la comunidad de HFMA en el espacio extraradical de las plantas de yuca en el experimento en campo. 
Comparando las comunidades de plantas inoculadas y plantas no inoculadas, la abundancia de morfotipos identificados como Rhizophagus (morfotipos 10, 15, 19, 24, 25, 34 y 35, Figura 1), no fue dominante en la comunidad, lo cual sugiere que el hongo inoculado no tiene un comportamiento invasivo que ponga en peligro la diversidad de los HFMA locales y además, que la comunidad local de HFMA fue resiliente a la inoculación.

\section{Efecto de la inoculación y de la fertilización fosfatada sobre la comunidad de HFMA}

El análisis de varianza mostró que la interacción entre la inoculación y la fertilización fosfatada tuvo un efecto significativo sobre los índices de diversidad $(p=0,017)$ y riqueza de morfotipos $(p=0,009)$ (Cuadro $2 a)$. Las comunidades inoculadas con una dosis de 50 \% de fertilización fosfatada, fueron más diversas y con mayor número de morfotipos, mientras que las comunidades inoculadas que no recibieron fertilizante fosfatado, fueron las menos diversas y con el menor número de morfotipos (Cuadro 2b).

Cuadro 2. Efecto de los tratamientos sobre la diversidad alfa de la comunidad de HFMA (a) Datos de valor $p$ para resultados del ANOVA en el experimento factorial (b) Medias para cada tratamiento, las letras indican las diferencias estadísticamente significativas según la prueba de Tukey.

\begin{tabular}{|l|l|c|c|c|}
\hline \multicolumn{1}{|c|}{ Variables } & Riqueza S' & Índice de Shannon H' & Índice de Pielou J' \\
\cline { 2 - 5 } a) & Inoculación & 0,201 & 0,248 & 0,663 \\
\cline { 2 - 5 } & Fertilización fosfatada & 0,078 & 0,093 & 0,521 \\
\cline { 2 - 5 } & Interacción de variables & 0,00 * $^{*}$ & $0,017^{*}$ & 0,811 \\
\hline \multirow{4}{*}{ Tratamiento } & Riqueza S' & Índice de Shannon H' & Índice de Pielou J' \\
\cline { 2 - 5 } b) & \multicolumn{1}{|c|}{ Sin inoculación 0\% P } & $21,00 \mathrm{ab}$ & $2,70 \mathrm{ab}$ & 0,88 \\
\cline { 2 - 5 } & Sin inoculación 50 \% P & $19,75 \mathrm{ab}$ & $2,66 \mathrm{ab}$ & 0,89 \\
\cline { 2 - 5 } & Sin inoculación. 100 \% P & $21,50 \mathrm{ab}$ & $2,78 \mathrm{ab}$ & 0,91 \\
\cline { 2 - 5 } & Con inoculación. 0\% P & $17,00 \mathrm{~b}$ & $2,47 \mathrm{~b}$ & 0,87 \\
\cline { 2 - 5 } & Con inoculación 50\% P & $24,50 \mathrm{a}$ & $2,87 \mathrm{a}$ & 0,90 \\
\cline { 2 - 5 } & Con inoculación 100\% P & $18,75 \mathrm{ab}$ & $2,61 \mathrm{ab}$ & 0,89 \\
\hline
\end{tabular}

En el escalamiento multidimensional no métrico, las comunidades de HFMA del tratamiento control - aquellas que no recibieron inoculación ni fertilizante fosfatado (No Inoculadas - 0 \% P) - se agruparon visiblemente separadas; así también las comunidades de HFMA que no recibieron fertilizante fosfatado, pero si fueron inoculadas (Inoculadas - 0 \% P) (Figura 3). Estas diferencias halladas con base en la presencia y número de morfotipos compartidos entre tratamientos, dan un indicio de la dinámica de estas comunidades y de la respuesta de estos hongos a los cambios en su entorno.

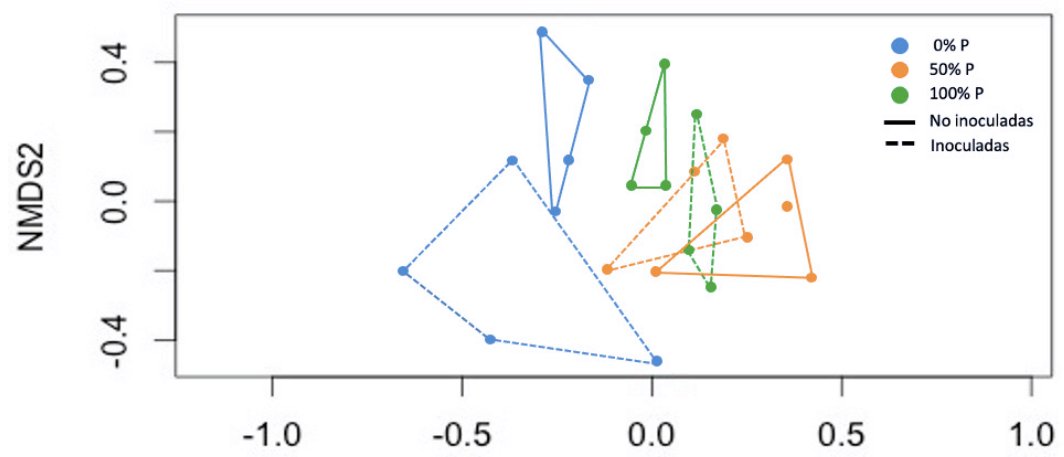

NMDS1

Figura 3. NMDS calculado para la comunidad de HFMA en la rizósfera de plantas de yuca, bajo los seis tratamientos en estudio. 
Los análisis multivariados, que detectan los cambios en el ensamblaje de las comunidades entre sitios (diversidad beta), mostraron diferencias significativas en la distribución de los morfotipos dentro de las comunidades de HFMA (PERMANOVA, $p<0,05$ ), con alteraciones a causa de la inoculación, la fertilización fosfatada y la interacción de estas variables (Cuadro 3 ).

Cuadro 3. Resultados del análisis PERMANOVA para las comunidades de HFMA presentes en la rizósfera de plantas de yuca, medida por medio de esporas. Valor $p$ con un alfa=0,05.

\begin{tabular}{|l|l|l|l|}
\hline \multicolumn{1}{|c|}{ Variable } & \multicolumn{1}{c|}{ Valor $\mathbf{F}$} & \multicolumn{1}{c|}{$\mathbf{R}^{2}$} & \multicolumn{1}{c|}{ Valor $\boldsymbol{p}$} \\
\hline Inoculación & 1,848 & 0,071 & $0,027^{*}$ \\
\hline Fertilización fosfatada & 2,0943 & 0,080 & $0,011^{*}$ \\
\hline Interacción de variables & 2,0133 & 0,07757 & $0,012^{*}$ \\
\hline
\end{tabular}

Por lo tanto, ocurrieron diferencias en la composición de las comunidades extraradicales de HFMA (diversidad beta), así también en la riqueza y diversidad, asociadas a la interacción de los tratamientos analizados en este estudio, aunque no existieron resultados estadísticamente significativos a nivel de uniformidad (diversidad alfa). Algunos estudios en comunidades de HFMA han mostrado correspondencia entre los resultados de la diversidad alfa cuando se comparan con los resultados de la diversidad beta ( Séry et al., 2018; Renaut et al., 2020). Sin embargo, resultados contrastantes entre diversidad alfa y beta han sido publicados previamente, sin que esto indique un resultado contradictorio. Por ejemplo, Peña-Venegas et al., (2019) estudió las comunidades de HFMA en suelos de la Amazonía colombiana y encontró que la variedad de las plantas de yuca no tuvo efecto significativo sobre los índices de diversidad alfa, sin embargo generó diferencias en la composición de las comunidades de HFMA, tanto en el espacio intra como extraradical.

\section{Efecto de la inoculación sobre las comunidades extra-radicales de HFMA}

Cuando se analiza la composición de las comunidades de HFMA a partir de las esporas presentes en el suelo rizosférico, es importante tener presente que las esporas de HFMA se consideran como estructuras de reposo y reservorios del material genético, las cuales pueden tener periodos de dormancia con diferente respuesta a la germinación, dependiendo del pH, el contenido de minerales, y la temperatura del suelo (Giovannini et al., 2020), además que no necesariamente representan la comunidad fisiológicamente activa en interacción con la planta. Por lo tanto, no es posible afirmar que los morfotipos que se describieron en la comunidad extraradical de las plantas de yuca, estaban en una relación simbiótica activa para el momento del muestreo; sin embargo, son una radiografía de aquellas especies que una vez presentes en la rizósfera, podrían colonizar al hospedero cuando las condiciones edáficas fuesen mas favorables. En este contexto, registrar los cambios de los diferentes géneros dentro de la comunidad extraradical, representa un paso hacia la comprensión de la respuesta de este grupo de hongos simbiontes ante el disturbio en suelos agrícolas del trópico, mas cuando se ha observado que la composición de la comunidad HFMA es específica de la planta y del suelo analizado (Renaut et al., 2020; Peña-Venegas et al., 2019).

En el presente estudio, se detectaron cambios en el ensamblaje de la comunidad de HFMA causados por la inoculación (Cuadro 3), lo cual refleja la respuesta de este grupo de organismos edáficos ante la presencia de un aislado foraneo. Aunque R. irregularis se ha sido descrito como un hongo ubicuo, y estudios de diversidad de HFMA en los suelos de la Orinoquía colombiana sugieren que esta especie es un habitante natural en los suelos analizados (Ordoñez et al., 2016), la inoculación con un hongo de alta variabilidad genética, se podría asemejar a la introducción de una especie diferente. Recientes resultados reportados por Savary et al. (2018) quienes analizaron los genomas de 81 aislados de $R$. irregularis provenientes de todo el mundo, establecen que existen al menos cuatro grupos genéticos principales dentro de la especie, y que la altísima diversidad intraespecífica ha sido subestimada y poco estudiada en el pasado. Adicional a este hecho, se ha evidenciado y reportado una alta diversidad funcional de $R$. 
irregularis. Diversidad funcional consiste en que, diferentes aislados de $R$. irregularis provocan una respuesta diferente en la misma especie vegetal (Ceballos et al., 2019). Es por lo tanto interesante estudiar, cómo es que esta diversidad funcional se extiende a la interacción con la comunidad de HFMA presente en el suelo y se convierte en un aspecto a tener en cuenta al momento de utilizar estos hongos como biofertilizantes.

Por otro lado, Hart et al. (2017) han propuesto que la intensidad de los cambios generados por el hongo inoculado, sobre la comunidad de HFMA residente, depende de la estrategia de vida del hongo inoculado y de los hongos presentes en el suelo. Por ejemplo, hongos con estrategia de vida tipo $r$, efectivos en colonizar nuevos ambientes, podrían crecer rápidamente en la rizósfera y desplazar a hongos residentes con estrategias de vida tipo K, aquellos que persisten en entornos con fuerte competencia por los recursos (Maherali and Klironomos, 2012; Powell and Rillig, 2018).

Sin embargo, a pesar de que $R$. irregularis, que por capacidad de colonización y velocidad de crecimiento, es considerado como un estratega tipo $r$, su comportamiento en este estudio no refleja un crecimiento invasivo en el espacio extra-radical 12 meses después de la inoculación. Las esporas de esta especie solo ocuparon en promedio el $12 \%$ del total de la comunidad, tanto para tratamientos inoculados como no inoculados (Figura 1). Futuros estudios, que tengan por objetivo comprobar el tipo de estrategia de un hongo HFMA y su efecto sobre la comunidad local de HFMA, deberán considerar hipótesis de exclusión o adaptación (Millar and Bennett, 2016) a una escala de especie, población y comunidad.

\section{Efecto combinado de la inoculación y la fertilización fosfatada sobre la estructura de las comunidades extra-radicales de HFMA}

Para entender, desde el contexto ecológico, los cambios que pudieron surgir por la inoculación y la fertilización fosfatada, los morfotipos fueron agrupados a nivel de género y sus abundancias fueron fusionadas para ser comparadas entre tratamientos (Figura 4). Para el género Glomus, la fertilización fosfatada causó una disminución en la abundancia relativa de esporas en la rizósfera $(p=0,0084)$. Previamente se ha reportado la disminución en la abundancia de Glomus como respuesta a la fertilización fosfatada (Alguacil et al., 2010). Se sugiere que esto ocurre, porque, cuando se aumenta la cantidad de minerales disponibles para las plantas, principalmente el P, se reduce el volumen de compuestos carbonatados que exudan las raíces para sostener la simbiosis micorrícica y como consecuencia se limita el crecimiento de algunas especies de la comunidad de HFMA (Kobae, 2019). Es allí donde las especies con mayor afinidad simbiótica serán las que permanezcan en la interacción.

También se encontró una diferencia en la abundancia relativa de Acaulospora en las comunidades inoculadas, siendo mas abundante con la inoculación ( $p=0,042$ ) (Figura 4c). Para los demás géneros no se encontraron diferencias significativas por efecto de la inoculación o la fertilización fosfatada (Figura 4a). Reportes previos han descrito al género Acaulospora como un estratega K, con una baja capacidad de colonizar las raíces (Maherali and Klironomos, 2012), siendo competidores débiles y sensibles al disturbio (De la Cruz-Ortiz et al., 2020). Esto se reflejó en los resultados aquí presentados, por cuanto la abundancia relativa de este género estuvo en promedio en el $3 \%$ del total de la comunidad extraradical. Estudios en suelos andinos, han reportado con sorpresa la presencia de una especie del género Acaulospora como la mas abundante en las raíces de cultivos de papa después de la inoculación con $R$. irregularis (Loján et al., 2017). Estas comparaciones son un reflejo de la reducida posibilidad de predicción sobre ensamblaje de las comunidades de HFMA, lo cual justifica el análisis de cada agroecosistema teniendo en cuenta la interacción suelo-planta-hongo. 
a)

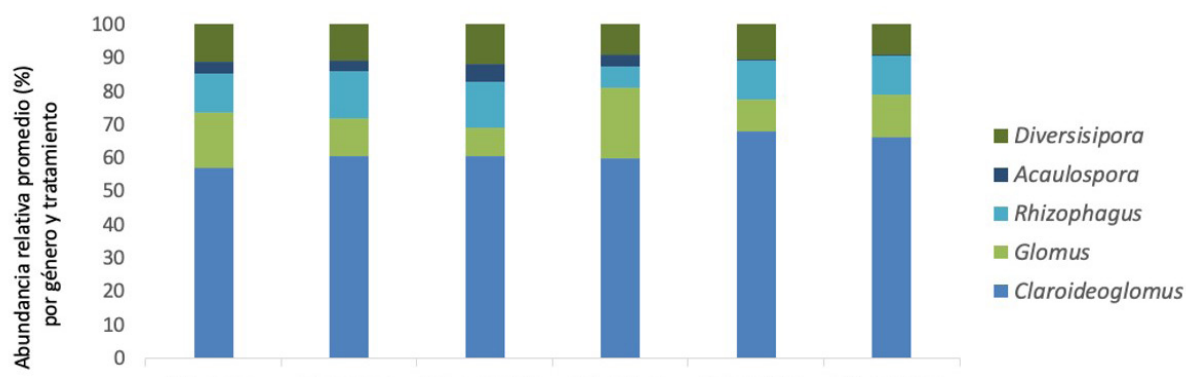
(1) $0 \% \mathrm{P}$
(2) $50 \% \mathrm{P}$
(3) $100 \% \mathrm{P}$
(4) $0 \% P$
(5) $50 \% \mathrm{P}$
(6) $100 \% P$
Con Inoculación
Sin Inoculación
Tratamientos

b)

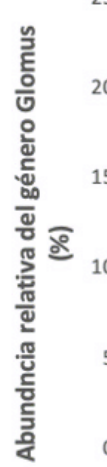

¿ 10

0

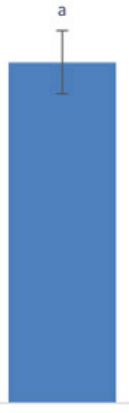

$0 \%$

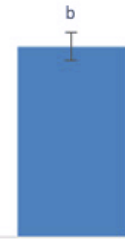

$50 \%$

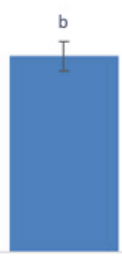

$100 \%$

Fertilización fosfatada aplicada al cultivo

c)
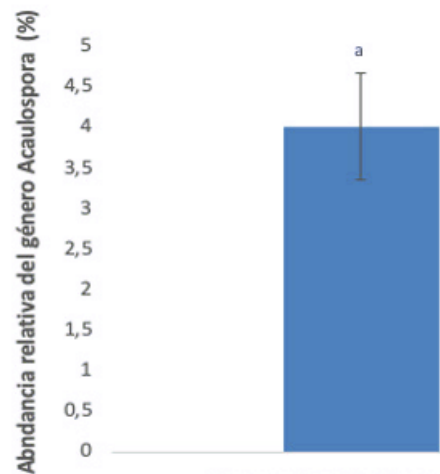

Comunidades inoculadas

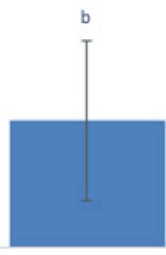

Comunidades no inoculadas

Figura 4. Abundancia relativa para los géneros que conformaron las comunidades extraradicales de HFMA doce meses después de aplicar los tratamientos a) Para todos los tratamientos. b) Género Glomus c) Género Acaulospora. Letras sobre las barras indican diferencias significativas para un alfa de 0,05. 


\section{Respuesta de la planta a la inoculación y la fertilización fosfatada}

Bajo este mismo experimento, la respuesta de las plantas de yuca a los tratamientos también fue evaluada. Se realizó la medición de variables como: peso fresco y seco de las raíces de yuca y el porcentaje de colonización intraradical. Los resultados se publicaron previamente por Ceballos et al. (2013) quienes reportaron un impacto positivo y estadísticamente significativo de la inoculación sobre el rendimiento del cultivo de yuca, siendo esa publicación una de las pioneras en demostrar la eficiencia de la inoculación con $R$. irregularis en experimentos en campo. Además, se reportó que los niveles de fertilización fosfatada interactúan con la inoculación e influyen en la respuesta de la planta, describiendo el tratamiento de inoculación y 50 \% P como el tratamiento que presentó mayor productividad de raíces de yuca en los suelos estudiados. De forma interesante, este mismo tratamiento presentó los mayores valores de diversidad y riqueza de morfotipos en este estudio.

Poco se ha explorado sobre la relación entre las comunidades de hongos micorrícicos que habitan en la rizósfera y los cambios fisiológicos en las plantas hospederas. Sin embargo algunos autores sugieren la posibilidad de un efecto directo o indirecto de la inoculación de HFMA en cultivos agrícolas (Rodriguez and Sanders, 2015). El efecto directo ejercido específicamente por la actividad del hongo inoculado, el cual deberá ser competitivo y eficiente traslocando nutrientes; y un efecto indirecto como consecuencia de los cambios en la comunidad local de HFMA causados por la inoculación. Debido a que los HFMA cumplen un papel esencial en la rizósfera, otra posibilidad de un efecto indirecto se presenta por los cambios generados sobre otras comunidades microbianas como consecuencia de la inoculación con $R$. irregularis y los cuales han sido reportados recientemente (Changey et al., 2019; Lammel et al., 2019). Sin embargo, estudios adicionales se requieren para identificar si los cambios en la comunidad de esporas detectados en este estudio, doce meses después de la inoculación, están relacionados con la respuesta de la planta.

\section{CONCLUSIONES}

Las comunidades de HFMA asociadas a las plantas de yuca en la Orinoquía Colombiana estuvieron conformadas por especies de los géneros Claroideoglomus, Glomus, Rhizophagus, Acaulospora y Diversispora. Claroideoglomus predominó en el espacio extraradical con el $62 \%$ de abundancia promedio en las muestras analizadas. La distribución de morfotipos de los géneros Acaulospora y Glomus, que habitaron el suelo rizosférico de las plantas de yuca de la variedad MCOL2737, estuvo afectada por la inoculación con R. irregularis y por la aplicación de fertilizante fosfatado.

Los resultados de esta investigación son importantes ya que demuestran que la cepa de $R$. irregularis empleada en este estudio, además de ser eficiente en términos de productividad del cultivo de yuca (Ceballos et al., 2013), también presentó un comportamiento deseable desde el punto de vista ecológico, sin generar cambios drásticos en la diversidad de la comunidad de HFMA locales. Posteriores estudios donde se analicen las comunidades de HFMA intra y extraradicales en cultivos de yuca podrán aportar información sobre el comportamiento de la comunidad de estos hongos, así como también en su relación con la productividad del cultivo.

\section{AGRADECIMIENTOS}

A la Swiss National Science Foundation por la financiación del proyecto "Cassava for food security and sustainability in Colombia: Biotechnological application of mycorrhizal fungi" y a la Universidad de la Salle, Sede Utopía, donde se estableció la parcela experimental. 


\section{REFERENCIAS}

ALEWELL, CHRISTINE; RINGEVAL, BRUNO; BALLABIO, CRISTIANO; ROBINSON, DAVID, A.; PANAGOS, PANO; BORRELLI, PASQUALE. Global phosphorus shortage will be aggravated by soil erosion. Nature Communications, v.11, n. 1, 2020, p. 4546. https://doi.org/10.1038/s41467-020-18326-7

ALGUACIL, MARIA DEL MAR; LOZANO, ZENAIDA; CAMPOY, MANUEL J.; ROLDAN, ANTONIO. Phosphorus fertilisation management modifies the biodiversity of am fungi in a tropical savanna forage system. Soil Biology and Biochemistry, v. 7, 2010, p. 1114-1122.

ALIYU, IBRAHIM A.; YUSUF, ADO A.; UYOVBISERE, EDWARD O.; MASSO, CARGELE; SANDERS, IAN R. Effect of co-application of phosphorus fertilizer and in vitro-produced mycorrhizal fungal inoculants on yield and leaf nutrient concentration of cassava. Plos One, v. 16, n. 6, 2019, e0218969. https://doi.org/10.1371/journal.pone.0218969

BONFANTE, PAOLA; VENICE, FRANCESCO. Mucoromycota: Going to the roots of plant-interacting fungi. Fungal Biology Reviews, v. 34, n. 2, 2020, p.100-113. https://doi.org/10.1016/j.fbr.2019.12.003

BRUNDRETT, MARK C.; TEDERSOO, LEHO. Evolutionary history of mycorrhizal symbioses and global host plant diversity. New Phytologist, v. 220, n. 4, 2018, p. 1108-1115. https://doi.org/10.1111/nph.14976

CABRALES-HERRERA, ELIECER-MIGUEL; BARRERA-VIOLETH, JOSÉ-LUIS; NOVOA-YANEZ, RAFAEL. Identification of Arbuscular Mycorrhizal Fungi in plantain producing municipalities in department of Cordoba, Colombia. Acta Agronómica, v. 67, n. 1, 2018, p. 17-22. http://dx.doi.org/10.15446/acag.v67n1.55854

CAMENZIND, TESSA; HEMPEL, STEFAN; HOMEIER, JÜRGEN; HORN, SEBASTIAN; VELESCU, ANDRE; WILCKE, WOLFGANG; RILLIG, MATTHIAS C. Nitrogen and phosphorus additions impact arbuscular mycorrhizal abundance and molecular diversity in a tropical montane forest. Global Change Biology, v. 20, n. 12,2014, p. 3646-3659. https://doi.org/10.1111/gcb.12618

CAMPO, SONIA; MARTÍN-CARDOSO, HÉCTOR; OLIVÉ, MARTA; PLA, EVA; CATALA-FORNER, MAR; MARTÍNEZ-EIXARCH, MAITE; SAN-SEGUNDO, BLANCA. Effect of root colonization by arbuscular mycorrhizal fungi on growth, Productivity and Blast Resistance in Rice. Rice, v. 13, n. 1, 2020, e42. https://doi.org/10.1186/s12284-020-00402-7

CEBALLOS, ISABEL; MATEUS, IVAN D.; PEÑA, RICARDO; PEÑA-QUEMBA, DIEGO-CAMILO; ROBBINS, CHANZ; ORDOÑEZ, YULI M.; ROSIKIEWICZ, PAWEL; ROJAS, EDWARD C.; THUITA, MOSES; MLAY, DEUSDEDIT-PETER; MASSO, CARGELE; VANLAUWE, BERNARD; RODRIGUEZ, ALIA; SANDERS, IAN R. Using variation in arbuscular mycorrhizal fungi to drive the productivity of the food security crop cassava. BioRxiv, 2019, 830547. https://doi.org/10.1101/830547

CEBALLOS, ISABEL; RUIZ, MICHAEL; FERNANDEZ, CRISTHIAN; PEÑA, RICARDO; RODRIGUEZ, ALIA; SANDERS, IAN R. The in vitro mass-produced model mycorrhizal fungus, Rhizophagus irregularis, significantly increases yields of the globally important food security crop cassava. Plos One, 2013, v. 8, n. 8, 2013, e70633. https://doi.org/10.1371/journal.pone.0070633

CHANGEY, FRÉDÉRIQUE; MEGLOULI, HACĖNE; FONTAINE, JOËL; MAGNIN-ROBERT, MARYLINE; TISSERANT, BENOIT; LERCH, THOMAS Z.; LOUNÈS-HADJ-SAHRAOUI, ANISSA. Initial microbial status modulates mycorrhizal inoculation effect on rhizosphere microbial communities. Mycorrhiza, v. 29, n. 5 , 2019, p. 475-487. https://doi.org/10.1007/s00572-019-00914-1 
COFRÉ, MARÍA-NOELIA; SOTERAS,FLORENCIA; IGLESIAS, MARÍA-DEL ROSARIO; VELÁZQUEZ, SILVANA; ABARCA, CAMILA; RISIO, LUCÍA; ONTIVERO, EMANUEL; CABELLO, MARTA N.; DOMÍNGUEZ, LAURA S.; LUGO, MÓNICA A. Biodiversity of arbuscular mycorrhizal fungi in South America: A review. Springer International Publishing, 2019, p. 4-72. https://doi.org/10.1007/978-3-030-15228-4_3

DE LA CRUZ-ORTIZ, ÁNGEL-VIVANI; LOPEZ-TELLOO, JONÁS-ÁLVAREZ; ROBLES, CELERINO; HERNÁNDEZCUEVAS, LAURA V. Tillage intensity reduces the arbuscular mycorrhizal fungi attributes associated with Solanum lycopersicum, in the Tehuantepec isthmus (Oaxaca), México. Applied Soil Ecology, v. 149, 2020,e103519. https://doi.org/10.1016/j.apsoil.2020.103519

ELLIOTT, ASHLEIGH J.; DANIELL, TIM J.; CAMERON, DUNCAN D.; FIELD, KATIE J. A commercial arbuscular mycorrhizal inoculum increases root colonization across wheat cultivars but does not increase assimilation of mycorrhiza-acquired nutrients. Plants, People, Planet, 2020, p. 1-12. https://doi.org/10.1002/ppp3.10094

FREW, ADAM. Contrasting effects of commercial and native arbuscular mycorrhizal fungal inoculants on plant biomass allocation, nutrients, and phenolics. Plants, People, Planet, 2020, p. 1-5. https://doi.org/10.1002/ppp3.10128

GARCÍA-DE-LEÓN, DAVID; VAHTER, TANEL; ZOBEL, MARTIN; KOPPEL, MATI; EDESI, LIINA; DAVISON, JOHN; AL-QURAISHY, SALEH; HOZZEIN, WAEL N.; MOORA, MARI; OJA, JANE; VASAR, MARTTI; ÖPIK, MAARJA. Different wheat cultivars exhibit variable responses to inoculation with arbuscular mycorrhizal fungi from organic and conventional farms. PloS One, v. 15, n. 5, 2020, e0233878. https://doi.org/10.1371/journal.pone.0233878

GIOVANNINI, LUCA; PALLA, MICHELA; AGNOLUCCI, MONICA; AVIO, LUCIANO ; SBRANA, CRISTIANA; TURRINI, ALESSANDRA; GIOVANNETTI, MANUELA. Arbuscular mycorrhizal fungi and associated microbiota as plant biostimulants: research strategies for the selection of the best performing inocula. Agronomy, v. 10, n. 1, 2020, p. 106. https://doi.org/10.3390/agronomy10010106

HART, MIRANDA M.; ANTUNES, PEDRO M.; CHAUDHARY, VEER-BALA; ABBOTT, LYNETTE K. Fungal inoculants in the field: is the reward greater than the risk?. Functional Ecology, v. 32, n. 1, 2017, p. 126-135. https://doi.org/10.1111/1365-2435.12976

HELGASON, THORUNN; DANIELL, TIM J.; HUSBAND, R.; FITTER, A.H.; YOUNG, J.P.W. Ploughing up the woodwide web?. Nature, v. 394, n. 6692, 1998, p. 431. http://dx.doi.org/10.1038/28764.http://dx.doi.org/10.1038/28764

HIJRI, MOHAMED. Analysis of a large dataset of mycorrhiza inoculation field trials on potato shows highly significant increases in yield. Mycorrhiza, v. 26, n. 3, 2016, p. 209-214. https://doi.org/10.1007/s00572-015-0661-4

INVAM, INTERNATIONAL CULTURE COLLECTION OF (VESICULAR) ARBUSCULAR MYCORRHIZAL FUNGI (INVAM). Extraction of Spores. 2021a.https://invam.wvu.edu/methods/spores/spore-extraction [Consultado Abril 06 de 2021].

INVAM, INTERNATIONAL CULTURE COLLECTION OF (VESICULAR) ARBUSCULAR MYCORRHIZAL FUNGI (INVAM). Trap culture. 2021b https://invam.wvu.edu/methods/culture-methods/trap-culture [Consultado Abril 06 de 2021].

JIN, HONGYAN; GERMIDA, JAMES J.; WALLEY, FRAN L. Suppressive effects of seed-applied fungicides on Arbuscular Mycorrhizal Fungi (AMF) differ with fungicide mode of action and AMF species. Applied Soil Ecology, v. 72, 2013, p. 22-30. https://doi.org/10.1016/j.apsoil.2013.05.013

KNERR, ANNE-JEAN'NE; WHEELER, DAVID; SCHLATTER, DAN; SHARMA-POUDYAL, DIPAK; DU-TOIT, LINDSEY J.; PAULITZ, TIMOTHY C. Arbuscular mycorrhizal fungal communities in organic and conventional onion crops in the Columbia basin of the pacific northwest United States. Phytobiomes Journal, v. 2, n. 4, 2018, p. 194-207.

https://doi.org/10.1094/PBIOMES-05-18-0022-R 
KOBAE, YOSHIHIRO. Dynamic phosphate uptake in arbuscular mycorrhizal roots under field conditions. Frontiers in Environmental Science, v. 6, 2019, e159. https://doi.org/10.3389/fenvs.2018.00159

$\mathrm{KOCH}$, ALEXANDER M.; ANTUNES, PEDRO M.; BARTO, E. KATHRYN; CIPOLLINI, DON; MUMMEY, DANIEL L.; KLIRONOMOS, JOHN N. The effects of arbuscular mycorrhizal (am) fungal and garlic mustard introductions on native am fungal diversity. biological invasions, v. 13, n. 7, 2011, p. 1627-1639. https://doi.org/10.1007/s10530-010-9920-7

KÖHL, LUISE; LUKASIEWICZ, CATHERINE E.; VAN DER HEIJDEN, MARCEL GA. Establishment and Effectiveness of Inoculated Arbuscular Mycorrhizal Fungi in Agricultural Soils. Plant, Cell and Environment, v. 39, 2016, p. 136-146. https://doi.org/10.1111/pce.12600

KRÜGER, MANUELA; KRÜGER, CLAUDIA; WALKER, CHRISTOPHER; STOCKINGER, HERBERT; SCHUSSLER, ARTHUR. Phylogenetic reference data for systematics and phylotaxonomy of arbuscular mycorrhizal fungi from phylum to species level. New Phytologist, v. 193, n. 4, 2012, p. 970-984. https://doi.org/10.1111/j.1469-8137.2011.03962.x

LAMMEL, D.R.; MEIERHOFER, D.; JOHNSTON, P.; MBEDI, S.; RILLIG, M.C. The effects of Arbuscular Mycorrhizal Fungi (AMF) and Rhizophagus irregularis in soil microorganisms accessed by metatranscriptomics and metaproteomics. BioRxiv, 2019, e860932. https://doi.org/10.1101/860932

LEE, JAIKOO; LEE, SANGSUN; YOUNG, J. PETER W. Improved PCR primers for the detection and identification of arbuscular mycorrhizal fungi. FEMS Microbiology Ecology, v. 65, n. 2, 2008, p. 339-349 https://doi.org/10.1111/j.1574-6941.2008.00531.x

LIN, CHUYU; WANG, YAOXIONG; LIU, MEIHUA; LI, QUAN; XIAO, WENFA; SONG, XINZHANG. Effects of nitrogen deposition and phosphorus addition on arbuscular mycorrhizal fungi of chinese fir (Cunninghamia lanceolata). Scientific Reports, v. 10, n. 1, 2020, e12260. https://doi.org/10.1038/s41598-020-69213-6

LOJÁN, PAUL; SENÉS-GUERRERO, CAROLINA; SUÁREZ, JUAN-PABLO; KROMANN, PETER; SCHÜßLER, ARTHUR; DECLERCK, STÉPHANE. Potato field-inoculation in ecuador with Rhizophagus irregularis: no impact on growth performance and associated arbuscular mycorrhizal fungal communities. Symbiosis, $v$. 73, n. 1, 2017, p. 45-56. https://doi.org/10.1007/s13199-016-0471-2

LÓPEZ-GARCÍA, ÁLVARO; JURADO-RIVERA, JOSÉ A.; BOTA, JOSEFINA; CIFRE, JOSEP; BARAZA, ELENA. Space and vine cultivar interact to determine the arbuscular mycorrhizal fungal community composition. Journal of Fungi, v. 6, n. 1, 2020, e317. https://doi.org/10.3390/jof6040317

MAHERALI, HAFIZ; KLIRONOMOS, JOHN N. Phylogenetic and trait-based assembly of arbuscular mycorrhizal fungal communities. Plos One, v. 7, n. 5, 2012, e36695. https://doi.org/10.1371/journal.pone.0036695

MARTIGNONI, MARIA M.; GARNIER, JIMMY; HART, MIRANDA M.; TYSON, REBECCA. Investigating the impact of the mycorrhizal inoculum on the resident fungal community and on plant growth. Ecological Modelling, v. 438, 2020, e109321.

https://doi.org/10.1016/j.ecolmodel.2020.109321

MASSA, N.; BONA, E.; NOVELLO, G.; TODESCHINI, V.; BOATTI, L.; MIGNONE, F.; GAMALERO, E.; LINGUA, G.; BERTA, G.; CESARO, P. AMF communities associated to Vitis vinifera in an italian vineyard subjected to integrated pest management at two different phenological stages. Scientific Reports, v. 10, n. 1, 2020, e9197. https://doi.org/10.1038/s41598-020-66067-w

MELO, CATARINA-DRUMONDE; WALKER, CHRISTOPHER; FREITAS, HELENA; MACHADO, ARTUR; BORGES, PAULO. Distribution of Arbuscular Mycorrhizal Fungi (AMF) in terceira and São Miguel Islands (Azores). Biodiversity Data Journal, v. 8, n. April, 2020, e49759.

https://doi.org/10.3897/BDJ.8.e49759 
MILLAR, NIALL S.; BENNETT, ALISON E. Stressed out symbiotes: Hypotheses for the influence of abiotic stress on arbuscular mycorrhizal fungi. Oecologia, v. 182, n. 3, 2016, p. 625-641. https://doi.org/10.1007/s00442-016-3673-7

MONTENEGRO, SANDRA-PATRICIA; BARRERA, SILVIA-EUGENIA; MOSQUERA, RAMÓN-ANTONIO. Occurrence of indigenous arbuscular mycorrhizal fungi associated with the rhizosphere of the naidí palm in Colombia. Ciencia y Tecnología Agropecuaria, v. 21, n. 3, 2020, e1275. https://doi.org/10.21930/rcta.vol21_num3_art:1275

OEHL, FRITZ; SIEVERDING, EWALD; PALENZUELA, JAVIER; INEICHEN, KURT; DA SILVA, GLADSTONEALVES. Advances in Glomeromycota taxonomy and classification. IMA Fungus, v. 2, n. 2, 2011, p. 191-199. https://doi.org/10.5598/imafungus.2011.02.02.10

OKSANEN, JARI; BLANCHET, F. GUILLAUME; KINDT, ROELAND; LEGENDRE, PIERRE;. MINCHIN, PETER R.; O'HARA, R.B.; SIMPSON, GAVIN L.; SOLYMOS, PETER; STEVENS, M. HENRY H.; WAGNER, HELENE. Vegan: Community Ecology Package. R Package Version 2.5-7. 2020. https://cran.r-project.org/web/ packages/vegan/vegan.pdf [Consultado Enero 07 de 2021].

ORDOÑEZ, YULI-MARCELA; VILLARD, LUCAS; CEBALLOS, ISABEL; MASCLAUX, FRÉDÉRIC G.; RODRIGUEZ, ALIA; SANDERS, IAN R. Inoculation with highly-related mycorrhizal fungal siblings, and their interaction with plant genoptypes, strongly shapes tropical mycorrhizal fungal community structure. BioRxiv, January, 2020, e230490. https://doi.org/10.1101/2020.07.31.230490

ORDOÑEZ, YULI-MARCELA. Dinámica de la comunidad de Hongos Formadores de Micorrizas Arbusculares después de inocular Rhizophagus irregularis en un sistema agrícola en el trópico [Tesis Doctorado en Biotecnología]. Bogotá (Colombia): Universidad Nacional de Colombia, Facultad de Ciencias, 2016, 50 p.

PEÑA-VENEGAS, CLARA P.; KUYPER, THOMAS W.; DAVISON, JOHN; JAIRUS, TEELE; VASAR, MARTTI; STOMPH, TJEERD-JAN; STRUIK, PAUL C.; MAARJA, ÖPIK. Distinct arbuscular mycorrhizal fungal communities associate with different manioc landraces and amazonian soils. Mycorrhiza, v. 29, n. 3, 2019, p. 263-275. https://doi.org/10.1007/s00572-019-00891-5

POWELL, JEFF R.; RILLIG, MATTHIAS C. Biodiversity of arbuscular mycorrhizal fungi and ecosystem function. New Phytologist, v. 220, n. 4, 2018, p. 1059-1075. https://doi.org/10.1111/nph.15119

R CORE TEAM. R: A Language and Environment for Statistical Computing. R Foundation for Statistical Computing, Vienna. 2018.http://www.R-project.org/ [consultado diciembre 10 de 2020].

RAMÍREZ-GÓMEZ, MARGARITA; PÉREZ-MONCADA, URLEY;SERRALDE-ORDOÑEZ, DIANA; PEÑARANDAROLÓN, ANDREA; ROVEDA-HOYOS, GABRIEL; RODRIGUEZ, ALIA. Diversity of arbuscular mycorrhizal fungi communities associated with cape gooseberry (Physalis peruviana I.) crops. Agronomía Colombiana,v. 37, n. 3, 2019, p. 239-254. http://dx.doi.org/10.15446/agron.colomb.v37n3.74008

RENAUT, SÉBASTIEN; DAOUD, RACHID; MASSE, JACYNTHE; VIALLE, AGATHE; HIJRI, MOHAMED. Inoculation with Rhizophagus irregularis does not alter arbuscular mycorrhizal fungal community structure within the roots of corn, wheat, and soybean crops. Microorganisms, v. 8, n. 1, 2020, e83. https://doi.org/10.3390/microorganisms8010083

RESTREPO-GIRALDO, KARLA-JAQUELINE; MONTOYA-CORREA, MARÍA-ISABEL; HENAO-JARAMILLO, PAULA; GUTIÉRREZ, LINA-ANDREA; MOLINA-GUZMÁN, LICET-PAOLA. Caracterización de hongos micorrízicos arbusculares de suelos ganaderos del trópico alto y trópico bajo en Antioquia, Colombia. Idesia, v. 37, n. 1, 2019, p. 35-44. http://dx.doi.org/10.4067/S0718-34292019005000301

RODRIGUEZ, ALIA; SANDERS, I.R. The role of community and population ecology in applying mycorrhizal fungi for improved food security. ISME Journal, v. 9, n. 5, 2015, p. 1053-1061. https://doi.org/10.1038/ismej.2014.207 
SANDERS, IAN.R.; ALT, MONICA; GROPPE, KATHLEEN; BOLLER, THOMAS; WIEMKEN, ANDREAS. Identification of ribosomal DNA polymorphisms among and within spores of the Glomales: Application to studies on the genetic diversity of arbuscular mycorrhizal fungal communities. The New Phytologist, v. 130, 1995, p. 419-427.

SAVARY, ROMAIN; MASCLAUX, FRÉDÉRIC G.; WYSS, TANIA; DROH, GERMAIN; CRUZ-CORELLA, JOAQUIM; MACHADO, ANA-PAULA; MORTON, JOSEPH B.; SANDERS, IAN R. A population genomics approach shows widespread geographical distribution of cryptic genomic forms of the symbiotic fungus Rhizophagus irregularis. The ISME Journal, v. 12, n. 1, 2018, p. 17-30. https://doi.org/10.1038/ismej.2017.153

SCHEMCK, N.Y.; PÉREZ, Y. Isolation and culture of VA Mycorrhizal Fungi. USA. McGraw-Hill Publishing Company, 1990, p. 237, ISBN 0070357013.

SCHUSSLER, ARTHUR. Glomeromycota species list. Glomeromycota: Phylogeny and Taxonomy of Glomeromycota (Arbuscular Mycorrhizal (AM) and Related Fungi). 2020. http://www.amf-phylogeny. com/ [Consultado enero 20 de 2021].

SENÉS-GUERRERO, CAROLINA; GIMÉNEZ, SALVADOR; PACHECO, ADRIANA; GRADILLA-HERNÁNDEZ, MISAEL-SEBASTIÁN; SCHÜßLER, ARTHUR. New MiSeq based strategy exposed plant-preferential arbuscular mycorrhizal fungal communities in arid soils of mexico. Symbiosis, v. 81, n. 3, 2020, p. 235-246. https://doi.org/10.1007/s13199-020-00698-5

SÉRY, DROLET-JEAN-MARC; VAN-TUINEN, DIEDERIK; DRAIN, ALICE; MOUNIER, ARNAUD; ZÉZÉ, ADOLPHE. The genus Rhizophagus dominates arbuscular mycorrhizal fungi communities in contrasted cassava field soils in Côte d'Ivoire. Rhizosphere, v. 7, 2018, p. 8-17. https://doi.org/10.1016/j.rhisph.2018.06.007

SONG, JUAN; YANYAN, HAN; BIANXIA, BAI; SHAN, JIN; QINGFANG, HE; JIAHONG, REN. Diversity of arbuscular mycorrhizal fungi in rhizosphere soils of the chinese medicinal herb Sophora flavescens ait. Soil and Tillage Research, v. 195, 2019, e104423. https://doi.org/10.1016/j.still.2019.104423

SOONVALD, LIINA; LOIT, KAIRE; RUNNO-PAURSON, EVE; ASTOVER, ALAR; TEDERSOO, LEHO. Characterising the effect of crop species and fertilisation treatment on root fungal communities. Scientific Reports, v. 10, n. 1, 2020, e18741. https://doi.org/10.1038/s41598-020-74952-7

STÜRMER, SIDNEY; KEMMELMEIER, KARL. The Glomeromycota in the Neotropics. Frontiers in Microbiology, v. 11, 2021, e553679. https://doi.org/10.3389/fmicb.2020.553679

THIOYE, BABACAR; VAN TUINEN, DIEDERIK; KANE, ABOUBACRY; DE FARIA, SERGIO-MANIA; NDIAYE, CHEIKH; DUPONNOIS, ROBIN; SYLLA, SAMBA-NDAO MUSTAPHA-BÂ; AMADOU. Tracing Rhizophagus irregularis isolate IR27 in Ziziphus mauritiana roots under field conditions. Mycorrhiza, v. 29, n. 1, 2019, p. 77-83. https://doi.org/10.1007/s00572-018-0875-3

VICTORINO, ÍRIS MARISA MAXAIEIE; VOYRON, SAMUELE; CASER, MATTEO; ORGIAZZI, ALBERTO; DEMASI, SONIA; BERRUTI, ANDREA; SCARIOT, VALENTINA; BIANCIOTTO, VALERIA; LUMINI, ERICA. Metabarcoding of soil fungal communities associated with alpine field-grown saffron (Crocus sativus l.) inoculated with AM fungi. Journal of Fungi, v. 7, n. 1, 2021, e45. https://doi.org/10.3390/jof7010045

VIEIRA, LARISSA-CARDOSO; ALVES DA SILVA, DANIELLE-KARLA, COSTA ESCOBAR, INDRA-ELENA; DA SILVA, JULYANA-MARIA; DE MOURA, INGRID-ANDRÊSSA; OEHL, FRITZ; ALVES DA SILVA, GLADSTONE. Changes in an arbuscular mycorrhizal fungi community along an environmental gradient. Plants, v. 9, n. 1, 2020, p. 1-16.

https://doi.org/10.3390/plants9010052 